\title{
Preface to HSOR special issue on instrumental variable methods
}

\author{
Sue M. Marcus • Naihua Duan
}

Published online: 23 November 2012

(C) Springer Science+Business Media New York 2012

Selection bias is a central issue for health services and outcomes research. In observational studies, individuals who receive a treatment often differ from those who do not. Even in randomized controlled trials, those who comply with the protocol often differ from those who do not. Within the field of causal inference, there are methods for adjusting for measured confounders; however, remaining bias due to unmeasured confounders and model misspecification is always a concern. About 10 years ago, Health Services \& Outcomes Research Methodology published a special issue on Causal Inference that was well-received in the field (Ash et al. 2001). The current special issue builds upon the previous special issue, with a focus on Instrumental Variable (IV) method, an approach for making causal inference about the effect of a treatment, even when there is unmeasured confounding. This is particularly attractive, as other causal methods such as propensity score matching can adjust only for observed characteristics and it is generally difficult to test whether hidden bias exists.

However, the validity of the IV approach relies upon a set of strong assumptions that may not hold perfectly in health services and outcomes research. Thus, the benefit of IV's ability to remove unmeasured confounding may be lost, at least in part, when these strong assumptions fail to hold. The four articles in this special issue (Baiocchi et al. 2012; Lu and Marcus 2012; Marcus et al. 2012; O’Malley 2012) focus on clarifying the IV assumptions and how to strengthen the causal inference when the IV assumptions fail to hold perfectly, as is often the case in 'real life' health services and outcomes research context.

O'Malley (2012), examine several longitudinal models and specifications of IV models with respect to underlying assumptions and resulting empirical consequences. The focus is on whether longitudinal data and assumptions can strengthen the IV inference. These methods are illustrated with data from a longitudinal study of mental health costs related to newer and more expensive second generation antipsychotics.

Baiocchi et al. (2012) present an innovative study design approach to IV called near/far matching. This approach is similar to propensity score matching; however, it can estimate causal effects when there is unmeasured confounding. Near/far matching is applied to

S. M. Marcus $(\bowtie) \cdot$ N. Duan

New York, NY, USA

e-mail: smarcus@nyspi.columbia.edu 
Medicare data to compare the effectiveness of carotid arterial stents with cerebral protection versus carotid endarterectomy for the treatment of carotid stenosis.

$\mathrm{Lu}$ and Marcus (2012) propose to combine both the IV and propensity score approaches for use in longitudinal randomized trials. This approach utilizes self-selection immediately after randomization as the instrument and carefully assesses whether the IV assumptions are satisfied in this case. Matching-enhanced IV estimation helps to control unmeasured founding by creating a subpopulation with similar covariate distributions but different selfselected treatment. This approach is illustrated using data from the Multi-modal Treatment study of children with Attention-deficit/hyperactivity Disorder, which was a 14-month randomized clinical trial, followed by an observational follow-up period.

Marcus et al. (2012) proposes to strengthen permutation inference for IV by incorporating full matching on the propensity score to examine the causal effect of an active ingredient within a randomized trial when there is noncompliance. This approach is illustrated with data from a study of homeless mentally-ill veterans who were randomized to case management versus case management plus a housing voucher; the goal is to estimate the causal effect of the proposed active ingredient, use of the housing voucher.

The IV method requires at least one valid instrument, a variable that is not associated with unmeasured confounding but which has no effect on the outcome other than its effect on the treatment. Finding a valid instrument can be tricky. The four articles in the special issue utilize a variety of instruments. Both O'Malley and Lu/Marcus articles make use of lagged variable or past treatment selection as the instrument in longitudinal studies. The Marcus and colleagues article uses randomization within the context of a randomized controlled trial as the instrument. The Baiocchi and colleagues article uses geographic variation as the instrument, a common approach for observational data.

A decade ago, the introduction to the HSOR Special Issue on Causal Inference (Ash et al. 2001) concluded that researchers seeking to draw causal inference from observational or quasi-experimental data should have "stated their assumptions clearly, and either assessed them, or discussed how they should be assessed." The current HSOR Special Issue takes this a step further by showing how IV inference can be strengthened by incorporating matching and longitudinal assumptions.

As we think about the past and current issues, we also have a vision for the next HSOR Special Issue on Causal Inference. We expect to see wider application of IV method in health services research in both observational and randomized studies, new and creative choices of instruments and matching approaches, and continued research into the similarities and differences between IV method and other causal mediation approaches.

\section{References}

Ash, A., Duan, N., Normand, S.-L.: HSOR special issue on causal inference: introduction. Health Serv. Outcomes Res. Method. 2(3/4), 165-167 (2001)

Baiocchi, M., Small, D., Polsky, D., Yang, L., Groeneveld, P.: Near/far matching-a study design approach to instrumental variables. Health Serv. Outcomes Res. Method. (2012)

Lu, B., Marcus, S.: Evaluating long term effects of a psychiatric treatment using instrumental variable and matching approaches. Health Serv. Outcomes Res. Method. (2012)

Marcus, S.M., Weaver, J., Lim, S., Duan, N., Gibbons, R.D., Rosenheck, R.: Assessing the causal effect of section 8 housing vouchers as the active ingredient for decreasing homelessness in veterans with mental illness. Health Serv. Outcomes Res. Method. (2012)

O'Malley, A.J.: Instrumental variable specifications and assumptions for longitudinal analysis of mental health cost offsets. Health Serv. Outcomes Res. Method. (2012) 